\title{
Predicting double-strand DNA breaks using epigenome marks or DNA at kilobase resolution
}

\author{
Raphaël Mourad ${ }^{1}{ }^{*}$, Krzysztof Ginalski $^{2}$, Gaëlle Legube ${ }^{3}$ and Olivier Cuvier ${ }^{1}$
}

\begin{abstract}
Double-strand breaks (DSBs) result from the attack of both DNA strands by multiple sources, including radiation and chemicals. DSBs can cause the abnormal chromosomal rearrangements associated with cancer. Recent techniques allow the genome-wide mapping of DSBs at high resolution, enabling the comprehensive study of their origins. However, these techniques are costly and challenging. Hence, we devise a computational approach to predict DSBs using the epigenomic and chromatin context, for which public data are readily available from the ENCODE project. We achieve excellent prediction accuracy at high resolution. We identify chromatin accessibility, activity, and long-range contacts as the best predictors.
\end{abstract}

Keywords: Double-strand breaks, Epigenetics, Chromatin, Machine learning

\section{Background}

Double-strand breaks (DSBs) arise when both DNA strands of the double helix are severed. DSBs are caused by the attack of deoxyribose and DNA bases by reactive oxygen species and other electrophilic molecules [1]. DSBs are particularly hazardous to a cell because they can lead to deletions, translocations, and fusions in the DNA, collectively referred to as chromosomal rearrangements [2]. DSBs are most commonly found in cancer cells. Several high-throughput sequencing techniques have been developed for the genome-wide mapping of DSBs in situ such as BLESS [3], GUIDE-seq [4], END-seq [5], and DSBCapture [6]. One of the most recent techniques, DSBCapture, was used to map more than 80000 endogenous DSBs at a resolution lower than $1 \mathrm{~kb}$ in human. To date, DSBs have been mapped at high resolution only for a few cell lines due to the high sequencing costs and experimental difficulties. This has prevented the comprehensive study of the DSB landscape in the human genome across diverse cell lines and tissues.

Chromatin immunoprecipitation followed by highthroughput DNA sequencing (ChIP-seq) and DNase I

\footnotetext{
*Correspondence: raphael.mourad@ibcg.biotoul.fr

${ }^{1}$ LBME, Centre de Biologie Intégrative (CBI), Université de Toulouse, CNRS, UPS,

118 , route de Narbonne, 31062 Toulouse, France

Full list of author information is available at the end of the article
}

hypersensitive site sequencing (DNase-seq) data are publicly available for dozens of cell lines and tissues from the ENCODE [7] and Roadmap Epigenomics [8] projects. On the one hand, recent studies have shown that the mapping of regulatory elements such as enhancers and promoters can be accurately predicted using available epigenome and chromatin data $[9,10]$. Other studies have shown that the epigenome can be predicted by combinations of DNA motifs and DNA shape [11-14]. On the other hand, DSBs and the resulting DNA repair mechanisms have been shown to be linked to epigenome marks, including H3K4me1/2/3 and chromatin accessibility [6]. Accordingly, PRDM9-mediated trimethylation of H3K4 (H3K4me3) was originally shown to play a critical role in regulating DSBs associated with meiotic recombination hotspots [15-17]. Moreover, the repair of DSBs involves both post-translational modification of histones, in particular $\gamma$-H2AX, and concentration of DNA-repair proteins at the site of damage $[18,19]$. It remains unclear to what extent DNA motifs or histone modifications predict or regulate the cellular response to DSBs in other developmental stages. Here, we thus sought to test whether publicly available epigenome and chromatin data, or DNA motifs and shape, could be used to predict DSBs.

In this article, we demonstrate, for the first time, that endogenous DSBs can be computationally predicted using 
the epigenomic and chromatin context, or using DNA sequence and DNA shape. Our predictions achieve excellent accuracy (area under the receiver operating characteristic curve or AUROC $>0.97$ ) at high resolution $(<1 \mathrm{~kb})$ using available ChIP-seq and DNase-seq data from public databases. Despite the highly imbalanced data when predicting DSBs genome-wide, our approach detects a reasonable number of false positives (area under the precision-recall curve or AUPR $=0.459)$. DNase, CTCF binding, and H3K4me1/2/3 are among the best predictors of DSBs, reflecting the importance of chromatin accessibility, activity, and long-range contacts in determining DSB sites and subsequent repairing. We also successfully predict DSB sites using DNA motif occurrences only $(\mathrm{AUROC}=0.839)$ and identify the CTCF motif as a strong predictor. In addition, DNA shape analysis further reveals the importance of the structure-based readout in determining DSB sites, complementary to the sequence-based readout (motifs).

\section{Results and discussion}

\section{Double-strand break prediction approach}

Our computational approach for predicting DSBs is schematically illustrated in Fig. 1. In the first step, we analyzed public DSBCapture data from Lensing el at. [6], which is the most sensitive and accurate genome-wide mapping of DSBs to date (Fig. 1a). DSBCapture captures
DSBs in situ and it can directly map them at singlenucleotide resolution. DSBCapture peaks were called with less than 1-kb resolution (median size of 391 bases). The DSBCapture peaks obtained from two biological replicates were intersected to yield more reliable DSB sites. Endogenous breaks were captured for normal human epidermal keratinocytes (NHEKs), for which numerous ChIP-seq and DNase-seq data are publicly available from the ENCODE project [7]. In the second step, we integrated and mapped different types of data within DSB sites and non-DSB sites. To prevent bias effects, non-DSB sites were randomly drawn from the human genome with sizes, GC, and repeat contents similar to those of DSB sites [20] (Fig. 1b). ChIP-seq and DNase-seq peaks in NHEKs, as obtained from the ENCODE project, were mapped to corresponding DSB and non-DSB sites [7]. We also mapped p63 ChIP-seq peaks from keratinocytes [21]. We further searched for potential protein-binding sites at DSB and non-DSB sites using motif position weight matrices from the JASPAR 2016 database [22], and predicted DNA shape at DSB and non-DSB sites using Monte Carlo simulations [23]. In the third step, a random forest classifier was built to discriminate between DSB sites and non-DSB sites based on epigenome marks or DNA (Fig. 1c). Random forest variable importance values were used to estimate the predictive importance of a feature. We also compared random forest predictions with another popular method,

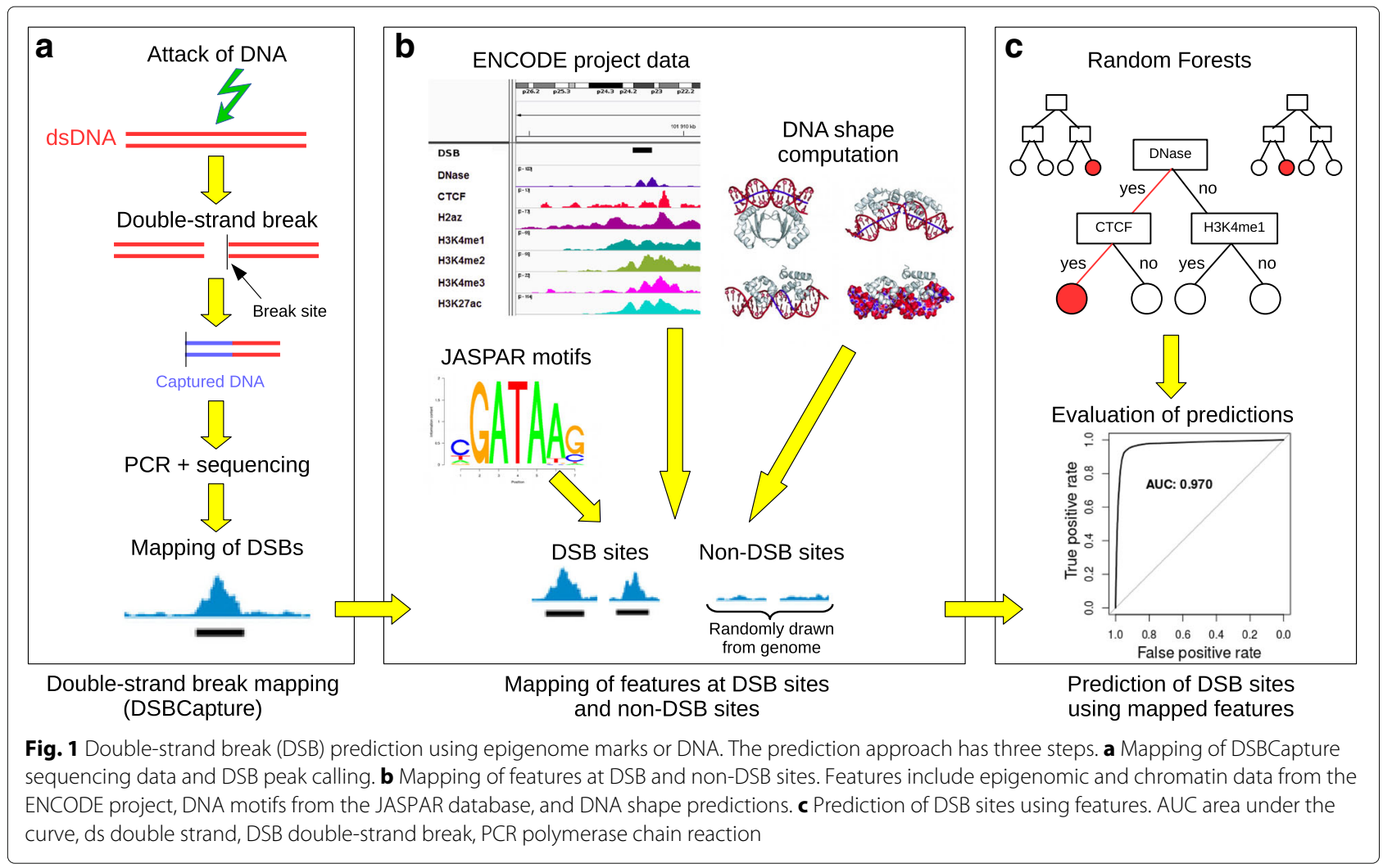


lasso logistic regression [24]. Using lasso regression, we assessed the positive, negative, or null contribution of a feature to DSBs. We then split the DSB dataset into a training set to learn model parameters by cross-validation, and into a testing set to compute the receiver operating characteristic (ROC) and precision-recall (PR) curves, as well as AUROC and AUPR, to evaluate prediction accuracy.

\section{Double-strand breaks are enriched with epigenome marks and DNA motifs}

We first sought to assess comprehensively the link between DSBs and epigenome marks or DNA motifs. As previously shown $[6,25]$, several epigenomic and chromatin marks colocalized at DSBs (Fig. 2a). Among the most enriched marks were DNase I hypersensitive sites, H3H4 methylation, and CTCF (Fig. 2b). For instance, $91 \%$ of DSBs colocalized to a DNase site, whereas this percentage dropped to $11 \%$ for non-DSB regions. This corresponded to an odds ratio (OR) of 89.3. Similarly, high enrichment was found for H3K4me2 (74\% versus 11\%; $\mathrm{OR}=22.4$ ) and for the insulator protein CTCF (25\% versus $2 \%$; OR = 19), which may involve its interactions with the insulator-related cofactor cohesin, which has been shown to protect genes from DSBs [26]. As such, DSBs mostly localized within open and active regions that were often implicated in long-range contacts [27]. Interestingly, DSBs also colocalized with tumor protein p63 binding $(19.4 \%$ versus $1 \%$; OR $=23.8)$, a member of the p53 gene family $[28,29]$. In addition, we could distinguish DNase and CTCF sites that were enriched at the center of DSBs from histone marks that were found at the edges of DSB sites (Fig. 2c). Therefore, the strong enrichment of epigenomic and chromatin marks at DSB sites suggests that DSB regions could be accurately predicted using available ChIP-seq and DNase-seq data from public databases, including ENCODE and Roadmap Epigenomics.

Previous enrichment analyses of DNA-binding proteins were limited by the ChIP-seq data available. Hence, we sought DNA motifs that may be enriched at DSB sites as a way to obtain a more comprehensive list of candidate DNA-binding proteins. Of the 454 available motifs from the JASPAR 2016 database, 134 were significantly enriched ( $p<0.05$, Bonferroni correction), indicating that DSBs were associated with a large number of proteinbinding sites (Fig. 2d). Among the most enriched and frequent motifs, we identified numerous motifs specifically recognized by protein cofactors of the transcription factor complex AP-1. This included JUND (OR = 1.40, $12 \%$ of DSBs), JUNB (OR $=1.27,19 \%$ of DSBs), the heterodimer BATF::JUN (OR = 1.31, 10\% of DSBs), and also FOS $(\mathrm{OR}=1.37,20 \%$ of DSBs), FOSL1 (OR $=1.37,17 \%$ of DSBs), and FOSL2 (OR $=1.27,18 \%$ of DSBs). Among the most enriched but less frequent motifs, we expectedly found CTCF (OR $=1.54,1.7 \%$ of DSBs), as well as members of the tumor protein family p53, i.e., p53 itself $(\mathrm{OR}=1.54,0.2 \%$ of DSBs), p63 (OR $=1.49,0.3 \%$ of DSBs), and p73 (OR $=1.54,0.1 \%$ of DSBs) $[28,29]$. Such enrichment of DNA motifs at DSB sites, therefore, supports that DNA sequence can alone predict some of the DSBs encountered.

\section{Prediction using epigenomic and chromatin data}

Given the strong link between DSBs and epigenomic and chromatin marks, we sought to build a classifier to discriminate DSB sites from non-DSB sites based on the presence or absence of such marks. For this, we used random forests, which are very efficient classifiers for predicting a feature. They can capture non-linear and complex interaction effects [30]. We split the data into a training set to learn model parameters and a testing set to evaluate prediction accuracy. Using this classifier, we obtained excellent predictions of DSBs based on the epigenomic and chromatin marks available $($ AUROC $=0.970$ and AUPR = 0.985; Fig. 3a; Additional file 1: Figure S1). Bootstrap analysis of 2000 replicates revealed that these predictions were very robust ( $95 \%$ confidence interval, CI, of AUROC: [0.968,0.972]). We also computed the variable importance (VI), which reflects the importance of a mark as a predictor (Fig. 3b). Among the marks, DNase showed the highest variable importance (VI $=0.180)$, reflecting the known higher chromatin accessibility after DNA damage [19] or the involvement of chromatin-remodeling complexes in DSB processing [31]. Other good predictors were CTCF $(\mathrm{VI}=0.042)$, p63 $(\mathrm{VI}=0.031)$, H3K4me1 $(\mathrm{VI}=0.028), \mathrm{H} 3 \mathrm{~K} 4 \mathrm{me} 2(\mathrm{VI}=0.019), \mathrm{H} 3 \mathrm{~K} 4 \mathrm{me} 3$ (VI = $0.012)$, and $\mathrm{H} 3 \mathrm{~K} 27 \mathrm{ac}(\mathrm{VI}=0.010)$, highlighting the roles of active chromatin, but also long-range contacts and DNA damage response in predicting DSB sites.

A drawback of variable importance lies in its inability to distinguish between the positive or negative contribution of the predictive mark on DSBs. For this reason, we also used lasso logistic regression to predict DSBs [24]. With this second model, we obtained excellent predictions, although slightly less accurate (AUROC $=0.967, \mathrm{CI}_{95 \%}$ : [0.966,0.971]; AUPR = 0.982; Additional file 1: Figure S2). From lasso regression, we could assess the positive or negative contributions of the predictive marks using beta coefficients (Fig. 3c). We also performed logistic regression without any regularization and obtained very similar coefficients (Additional file 1: Figure S3). This allowed us to compute $p$ values associated with the coefficients. We found that all variables, except H3K79me2, H3K9ac, and H4K20me1, were significantly associated with DSBs (Additional file 1: Table S1). We identified positive predictive contributions of DNase, CTCF, p63, H3K4me1, and H3K4me2 marks, as previously revealed by enrichment analysis. We also uncovered negative predictive contributions of H3K9ac, H3K36me3, and H3K79me2. 


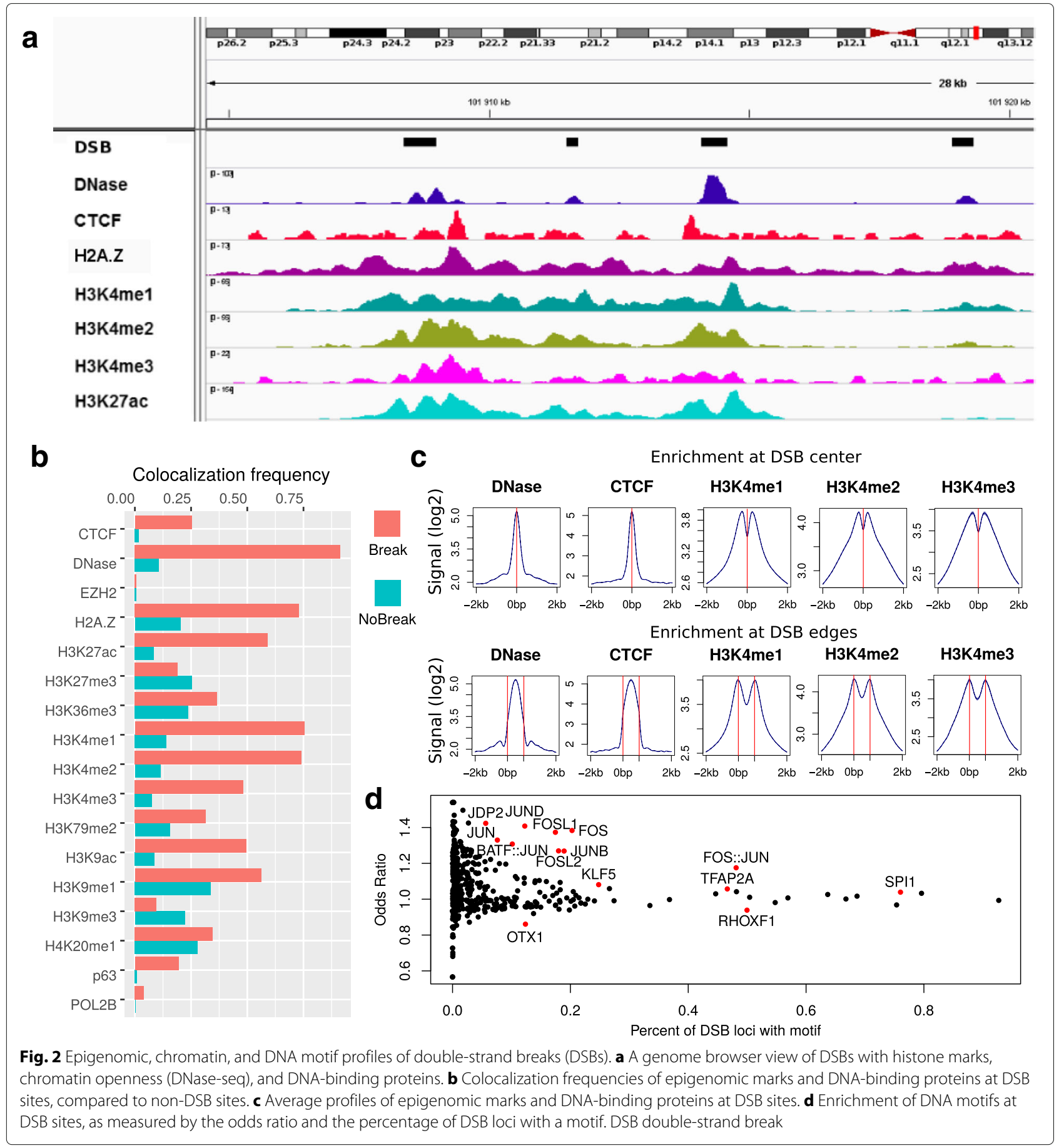

In agreement, $\mathrm{H} 3 \mathrm{~K} 9 \mathrm{ac}$ was shown to be rapidly and reversibly reduced in response to DNA damage [32]. Moreover, H3K36me3 may negatively impede DSBs by restricting chromatin accessibility through nucleosome positioning [33] or more directly by favoring the repair of DSBs [34].

We next sought to build a classifier using only one or two epigenomic marks, because this may be able to predict DSB sites even for cells for which only a few data points are available. We found that DNase I sites alone were sufficient to achieve good prediction accuracy $($ AUROC $=$ 0.919 and AUPR $=0.962$; Fig. 3d; Additional file 1: Figure S4), whereas H3K4me2 was not sufficient $($ AUROC $=0.816$ and AUPR = 0.907; Fig. 3d; Additional file 1: Figure S4). Combinations of DNase with H2A.Z or H3K4me1 yielded very accurate predictions $(A U R O C=0.952$ and $A U P R=$ 0.977; AUROC $=0.951$ and $\mathrm{AUPR}=0.976$, respectively; Fig. 3d; Additional file 1: Figure S4), close to the model 


\section{a}

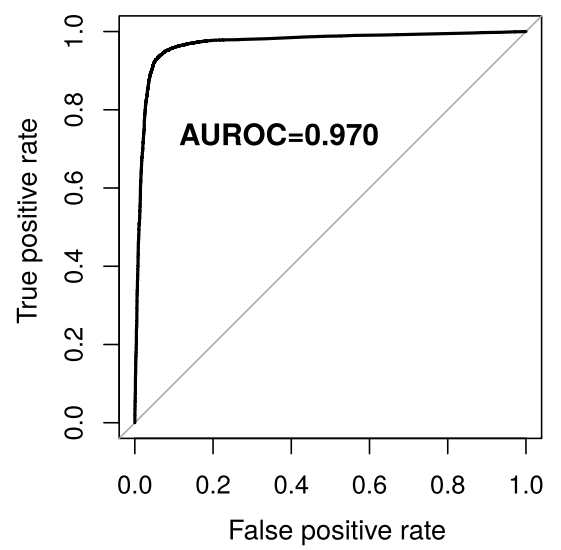

C

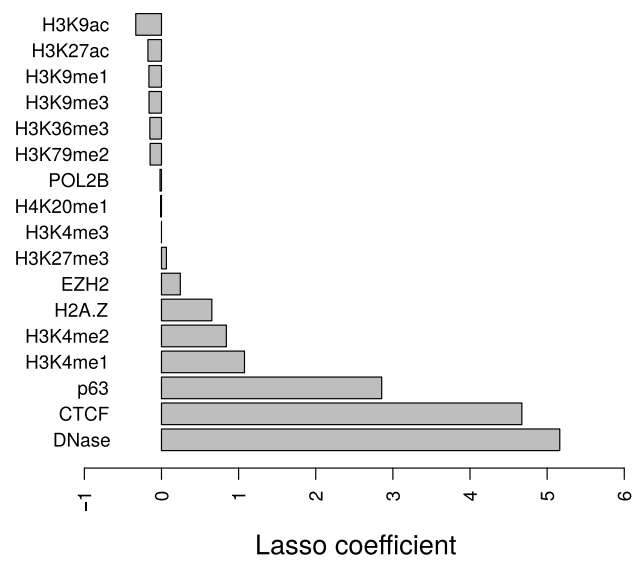

b

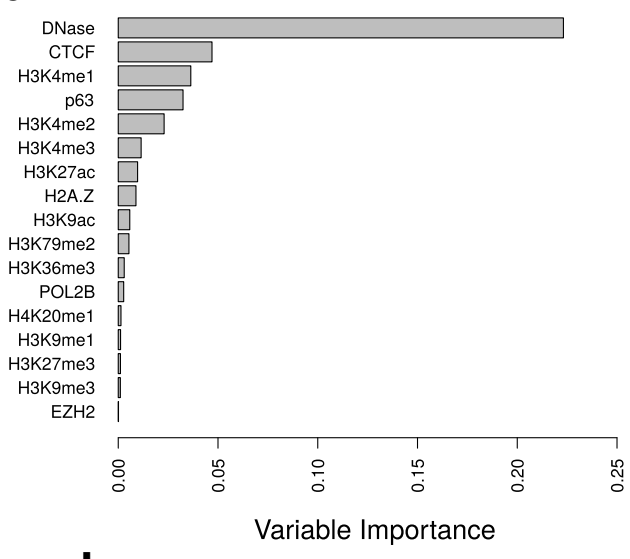

d

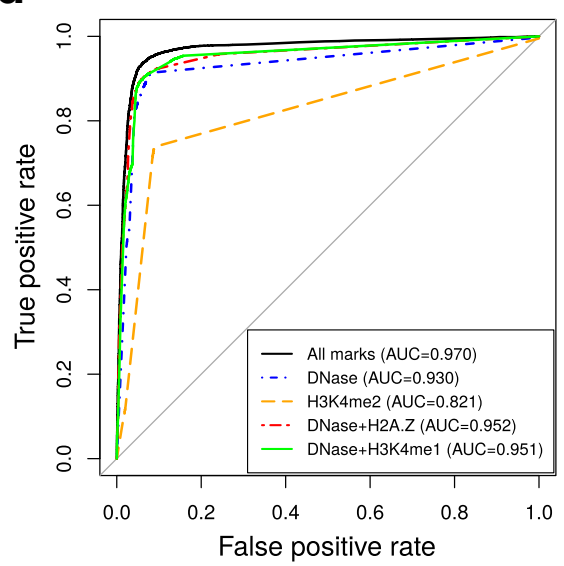

Fig. 3 Prediction of double-strand breaks using epigenomic and chromatin data with random forests. a Receiver operating characteristic curve for the prediction of double-strand breaks. Area under the ROC curve (AUROC) is plotted. $\mathbf{b}$ Variable importance of epigenomic and chromatin variables. c Lasso logistic regression coefficients. d Different predictive models including all variables, DNase only, H3K4me2 only, DNase+H2A.Z, or $\mathrm{DNase}+\mathrm{H} 3 \mathrm{~K} 4 \mathrm{me} 1$. AUROC area under the receiver operating characteristic curve

including all marks. Because DNase was a strong predictor, we explored where DNase was absent at DSBs to identify other marks that could be predictive here. We thus built a classifier using only DSBs that did not overlap any DNase site. DSB sites were still predicted well (AUROC $=0.869$ and AUPR $=0.792$; Additional file 1: Figure S5a and S5b), and CTCF and H3K4me1 were the most highly predictive variables (Additional file 1: Figure S5c). This revealed enhancer looping as a major driver of DSBs, in agreement with recent studies showing that DSBs form at loop anchors [35] and that CTCF facilitates DSB repair [36]. These results demonstrate that DSBs can be accurately predicted at less than $1-\mathrm{kb}$ resolution using just a small amount of data.

\section{Comparison with BLESS experiment and validation using an independent dataset}

We then compared previous DSB predictions with DSBs identified by BLESS experiments $[3,6]$. We also included in the comparison DSBCapture DSBs as the gold standard because of its higher sensitivity compared to BLESS: 84821 DSBs were found by DSBCapture compared to 18510 DSBs found by BLESS [6]. We first looked at predicted DSB sites surrounding the two genes MYC and MAP2K3 (Fig. 4a). For MYC, random forests correctly identified the four DSBs that were detected by DSBCapture, but erroneously predicted one DSB (yellow circle), whereas BLESS identified only one DSB out of four. For MAP2K3, random forests successfully predicted all DSBs detected by DSBCapture, whereas BLESS identified only three DSBs out of 11 .

We then compared predictions with BLESS at the genome-wide level (Fig. 4b). We observed that random forests correctly predicted 18084 out of 18510 DSB sites (97.70\%) found by BLESS, while it also successfully identified an additional 63587 out of 66591 DSB sites (95.49\%) found by DSBCapture that were not detected by BLESS. The model misclassified only 1552 out of 83225 predicted 

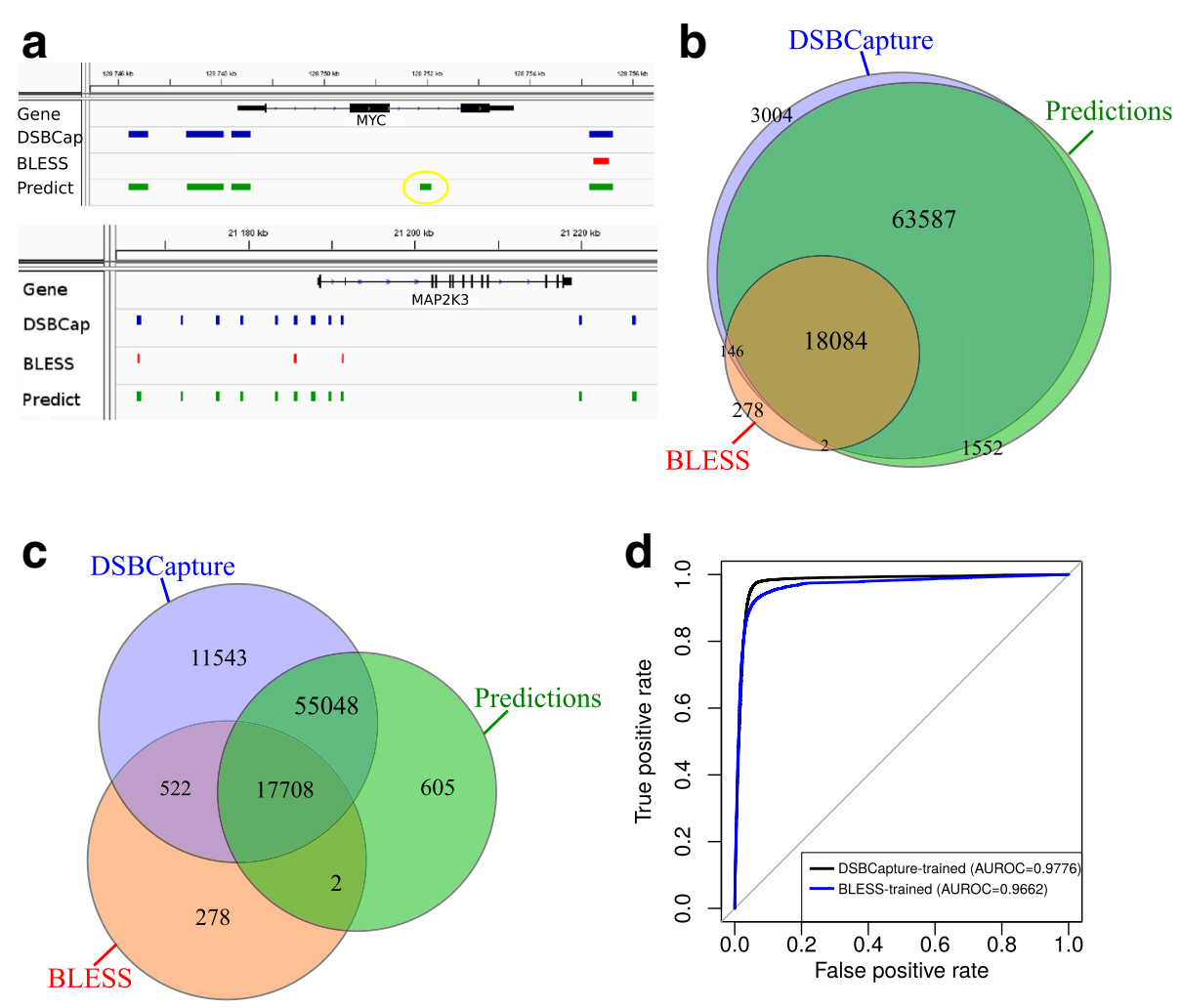

e
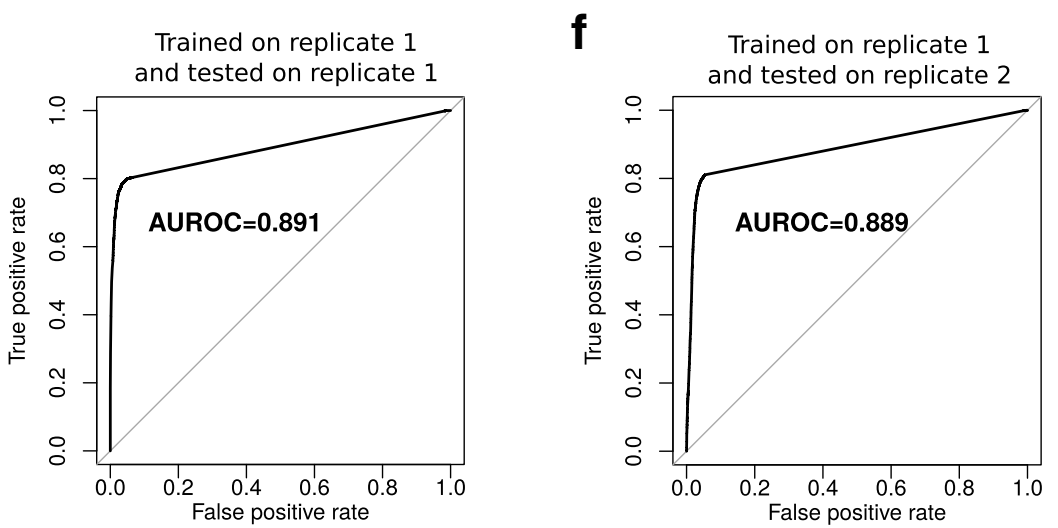

Fig. 4 Comparison of predicted and BLESS double-strand breaks (DSBs) and validation with an independent dataset. a Comparison for the MYC and MAP2K3 genes. b Venn diagram illustrating the overlaps between DSBCapture, random forest DSBCapture-trained model predictions, and BLESS DSBs. $\mathbf{c}$ Venn diagram illustrating the overlaps between DSBCapture, random forest BLESS-trained model predictions, and BLESS DSBS. $\mathbf{d}$ Comparison of receiver operating characteristic (ROC) curves between DSBCapture-trained and BLESS-trained models. Areas under the ROC curves (AUROCs) are plotted. e ROC curve for the prediction of DSBs trained on replicate 1 and tested on the same replicate. $\mathbf{f}$ ROC curve for the prediction of DSBs trained on replicate 1 and tested on replicate 2. AUROC area under the ROC curve, DSB double-strand break, ROC receiver operating characteristic

DSB sites (1.86\%). However, this previous prediction comparison should be carefully interpreted, because the model was learned from DSBCapture and then used to predict DSBCapture and BLESS DSBs.

To demonstrate the power of model-based predictions further, we devised another computational experiment, which consisted of training the model with BLESS DSBs and then predicting DSBCapture DSBs to test if the model could predict DSBCapture DSBs that were not detected by BLESS. Very interestingly, we found that the model was able to predict an additional 55048 out of 84821 DSBs (64.90\%) that were detected by DSBCapture but not by BLESS, and it identified only 605 DSBs out of 73363 predicted DSBs $(0.82 \%)$, which may be false positives not detected by DSBCapture and BLESS (Fig. 4c).

We then sought to compare models learned using DSBCapture and BLESS DSBs with a fair benchmark. For this, we devised the following strategy. A first model was 
learned from DSBCapture and was used to predict BLESS DSB sites (the DSBCapture-trained model), and a second model was learned from BLESS and was used to predict DSBCapture DSB sites (the BLESS-trained model). We found that both models had very good prediction performance $\left(\right.$ AUROC $C_{\text {model } 1}=0.9776$ and $\mathrm{AUPR}_{\text {model }}=$ 0.971; AUROC $_{\text {model } 2}=0.9662$ and $\mathrm{AUPR}_{\text {model } 2}=0.983$; Fig. 4d; Additional file 1: Figure S6).

In the previous section, we evaluated the accuracy of model predictions using a testing dataset that was from the same data as the training data (DSBs that overlapped between two replicates were split into a training dataset and a testing dataset). Here, we assessed model predictions by training random forests on one biological replicate and by testing prediction accuracy on a second biological replicate. For this, we used the two available DSBCapture biological replicates [6]. Accordingly, we used ENCODE epigenomic and chromatin data for which two biological replicates were available: DNase, CTCF, H3K4me3, H3K27me3, and H3K36me3. The first (respectively, second) replicates of the ENCODE data were associated with the first (respectively, second) DSBCapture replicate. Using only those five DNase-seq and ChIP-seq items, the model that was learned with the first replicate achieved accurate predictions on the testing data from the first replicate $($ AUROC $=0.891$ and AUPR $=$ 0.906; Fig. 4e; Additional file 1: Figure S7a). Note that the observed lower accuracy compared to that in the previous section (Fig. 3a,d) can be explained by the small amount of available epigenomic and chromatin data, and the lower reliability of DSBs identified using only one DSBCapture replicate. To validate the model on an independent dataset, we predicted DSBs from the second replicate using the model trained on the first replicate together with DNase-seq and ChIP-seq data for the second replicate. We obtained accurate predictions close to that obtained for the first replicate (AUROC $=0.889$ and $A U P R=0.913$; Fig. 4f; Additional file 1: Figure S7b). These accurate predictions demonstrate that using a classifier trained with epigenome and chromatin data is a reliable strategy for predicting DSBs.

\section{The impact of controls on prediction}

To assess if the high predictive accuracy of the model was inflated due to the way we selected non-DSB sites (the negative class), we devised different strategies. We first focused on gene promoters and built a random forest classifier to discriminate between promoters with DSBs (16801 sites) and promoters without (48 838 sites). As previously done, we computed the ROC curve but we also included the PR curve to account for class imbalance. We obtained very good performance for both the ROC curve (AUROC $=0.941$; Fig. $5 \mathrm{a})$ and the PR curve $(A U P R=$ 0.860; Fig. 5b). Second, we built a classifier to discriminate between gene bodies with DSBs (2187 sites) and gene bodies without (34573 sites). We also obtained a very good ROC curve (AUROC $=0.943$; Fig. $5 \mathrm{c}$ ), but with a lower PR curve because of the higher class imbalance in gene bodies (AUPR $=0.538$; Fig. $5 \mathrm{~d}$ ). Third, we built a classifier to discriminate between enhancers with DSBs (7373 sites) and enhancers without (38521 sites). We again observed a very good ROC curve (AUROC $=0.933$; Fig. $5 \mathrm{e}$ ) and good PR (AUPR = 0.705; Fig. 5f). Fourth, we evaluated predictions over the whole genome in an unbiased way. For this, we split the genome into 250-base bins. Then we built a classifier to discriminate between bins with DSBs (189 132 bins) and bins without (11 362262 bins). Using this approach, we obtained very good ROC accuracy (AUROC $=0.967)$ but with lower PR accuracy (AUPR $=0.459$ ) due to the high class imbalance, revealing a high number of false positives detected genome-wide by our method. We concluded that the excellent accuracy of model-based predictions was not inflated due to the way non-DSB sites were selected over the genome.

\section{Prediction in another cell type}

To validate our model-based predictions further, we used the random forest learned from DSBs in one cell type (NHEK) to predict DSBs in another cell type (U2OS). For this, we used data that were available for both NHEK and U2OS cells: DNA-seq, CTCF, H3K4me1/3, H3K9me3, H3K27ac, H3K27me3, H3K36me3, and POL2B. The validation is illustrated in Additional file 1: Figure S8. In summary, we trained a random forest with DSBCapture DSBs and DNase-seq and ChIP-seq data in NHEKs. We then predicted DSBs in U2OS cells using the NHEKtrained random forest with U2OS DNA-seq and ChIP-seq data. We validated the predictions with U2OS DSB data.

To evaluate prediction accuracy, we used the DSB data (DSBCapture [6] and BLESS [37]) that were generated for a specific cell line called U20S AID-DIvA. These DSB data were the only ones available in U20S. This cell line was a U2OS cell line that expressed the AsiSI restriction enzyme inducing DSBs at targeted sites [38]. To focus on endogenous DSBs, we kept only DSB data that did not overlap AsiSI sites. Most likely, only a fraction of all endogenous DSBs in U2OS could be mapped because DSB read coverage was low outside AsiSI sites.

In the first benchmark, we computed ROC and PR curves to evaluate the accuracy of model-based predictions. We compared our DSB predictions to a list of 2327 DSB sites identified by DSBCapture peak calling and 6443 non-DSB sites that were randomly drawn. Although this endogenous DSB list was far from complete, we obtained good prediction accuracy $($ AUROC $=0.835$; $\mathrm{Cl}_{95 \%}$ : $\left.0.824,0.846\right]$; AUPR $=0.881$; Fig. 6a; Additional file 1: Figure. S9). In agreement, we found that U2OS DSB prediction using a U2OS-trained random forest 


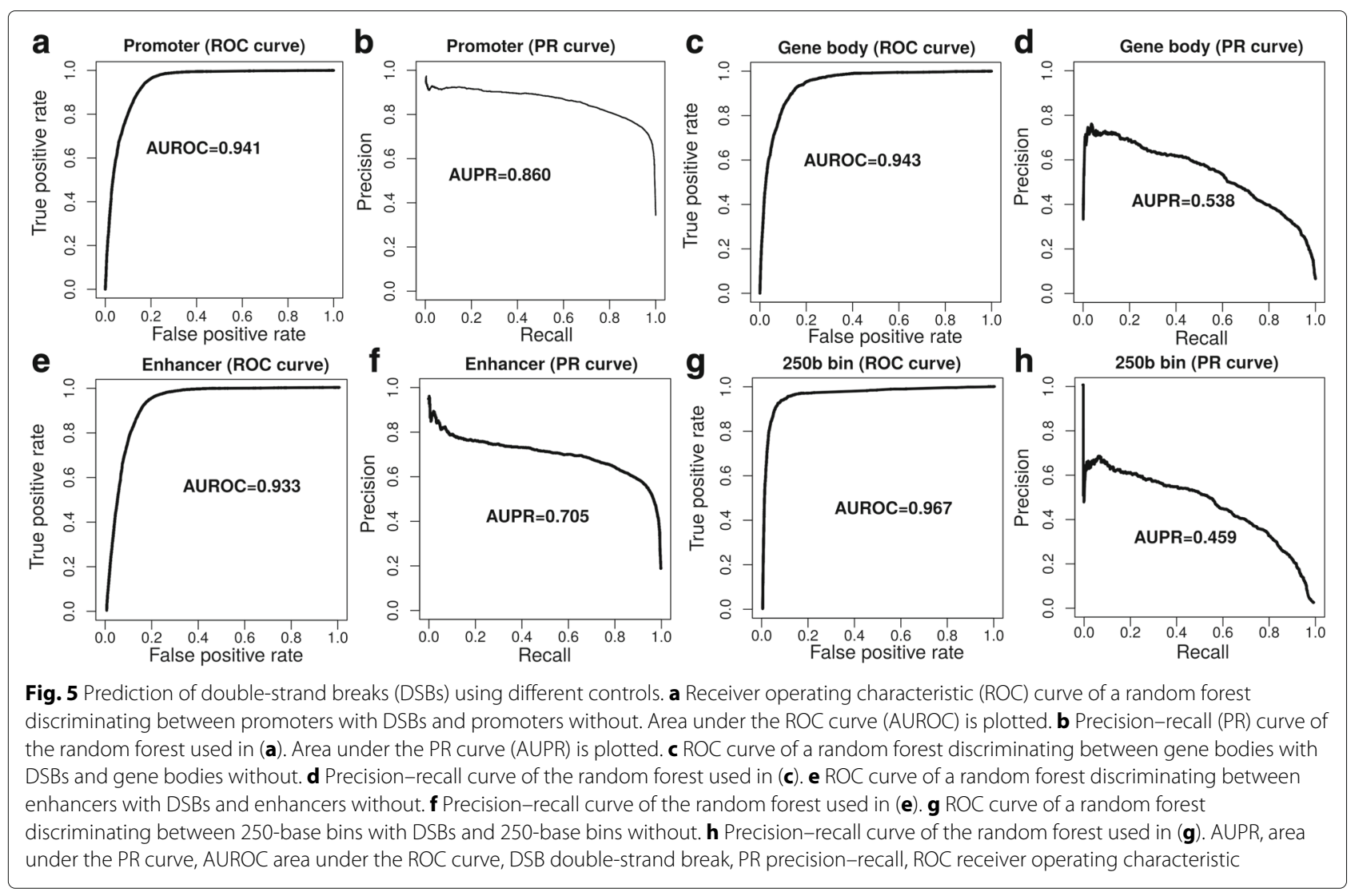

yielded only slightly better predictions than using a NHEK-trained random forest $\left(\right.$ AUROC $=0.859 ; \mathrm{CI}_{95 \%}$ : $[0.849,0.868] ;$ AUPR $=0.904$; Additional file 1: Figure S10). Moreover, DNase and CTCF had the highest variable importance, as found in NHEKs (Fig. 6b). Unfortunately, we could not carry out the same ROC and PR curve analyses with the BLESS data because not enough DSB sites were identified by peak calling.

In the second benchmark, we split the genome into 250-base bins and then predicted DSBs genome-wide. The model identified 87190 bins with a high DSB score (predicted DSBs) and 77510 bins with a low DSB score (predicted controls). As expected, we found a high enrichment of both DSBCapture and BLESS reads at predicted DSBs compared to predicted controls (Fig. 6c). On average, both DSBCapture and BLESS signals accordingly increased with the predicted DSB signal (Additional file 1: Figure S11a,b). Fortunately, there were also ChIP-seq data available for XRCC4, a DNA repair protein involved in non-homologous end-joining. Hence, we looked at whether XRCC4 was recruited at predicted DSBs. We found a high enrichment of XRCC4 at predicted DSBs compared to predicted controls (Fig. 6c), and an increase of the XRCC4 signal depending on the predicted DSB signal (Additional file 1: Figure S11c). In addition, ChIP-seq data were available for $\gamma-\mathrm{H} 2 \mathrm{AX}$, a histone mark that is induced at a megabase domain scale after DSBs, but is depleted on the few kilobases surrounding the exact break point $[38,39]$. Accordingly, we observed that $\gamma$-H2AX was depleted at predicted DSBs compared to predicted controls (Fig. 6c), and we found a decrease of the $\gamma-\mathrm{H} 2 \mathrm{AX}$ signal with the predicted DSB signal (Additional file 1: Figure S11d).

Additionally, we performed genome-wide DSB predictions in two other cell types for which endogenous DSB data were available, namely KBM7 (chronic myelogenous leukemia) and MCF-7 (breast cancer). For KBM7 cells, we used DNase-seq, CTCF, H3K4me1/me3, and H3K9me3 for prediction and BLISS for validation [40]. The model identified 163113 bins with a high DSB score (predicted DSBs) and 115204 bins with a low DSB score (predicted controls). We found an enrichment of BLISS reads at predicted DSBs compared to predicted controls (Additional file 1: Figure S12a). On average, the BLISS signal accordingly increased with the predicted DSB signal (Additional file 1: Figure S12b). For MCF-7 cells, we used DNase-seq, CTCF, H3K4me1/me3, H3K9ac/me3, and H3K27me3 for prediction and END-seq for validation [35]. The model identified 54746 bins with a high DSB score (predicted DSBs) and 84576 bins with a low DSB score (predicted controls). As expected, we found an enrichment of END-seq reads at predicted DSBs compared to predicted 

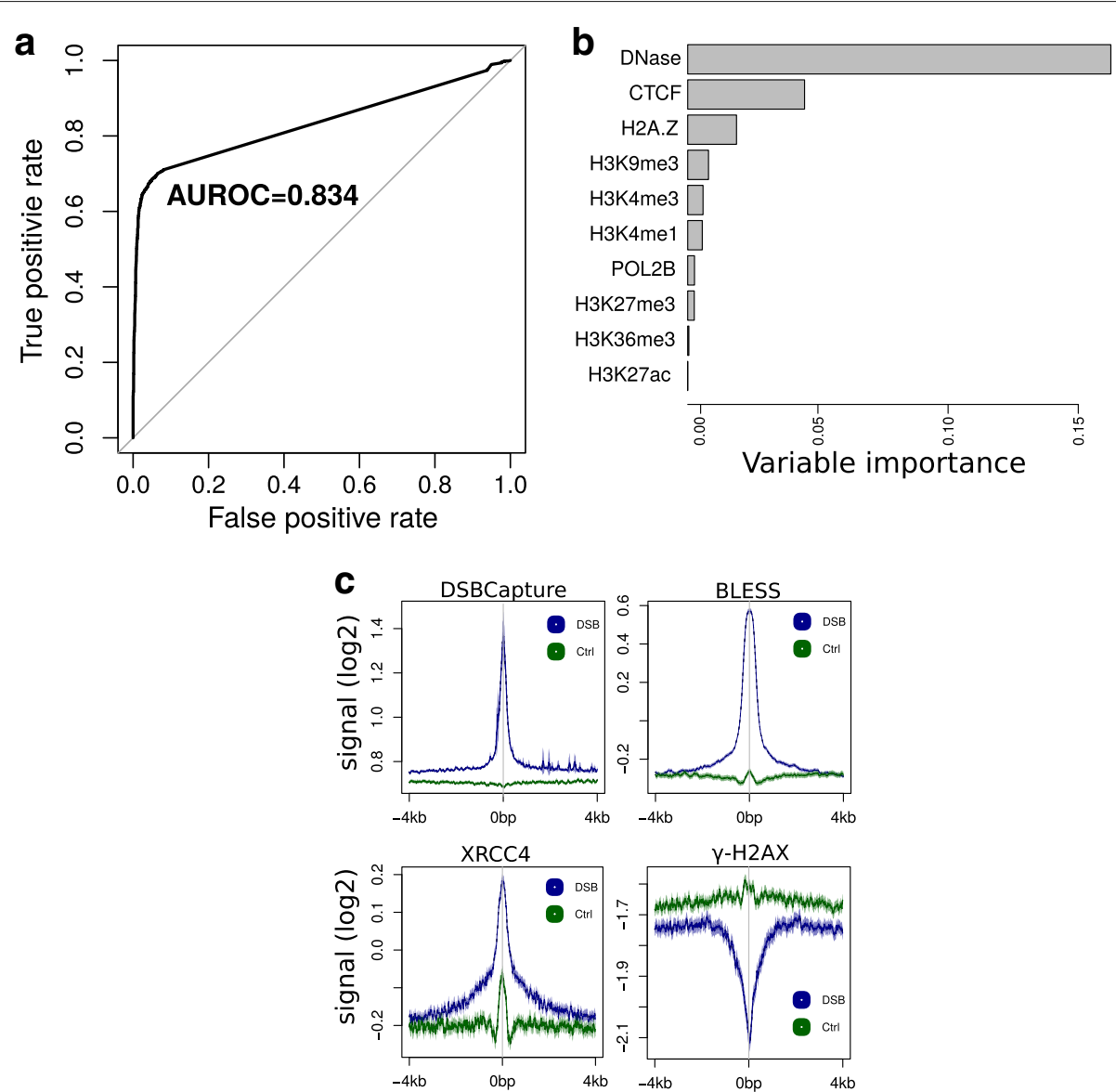

Fig. 6 Prediction of double-strand breaks (DSBs) using a random forest learned from DSBs in one cell type (NHEK) to predict DSBs in another cell type (U2OS). a Receiver operating characteristic (ROC) curve to predict U2OS DSBs using the NHEK-learned random forest. Area under the ROC curve (AUROC) is plotted. $\mathbf{b}$ Variable importance from the prediction of U2OS DSBs using the U2OS-learned random forest. c Average profiles of DSBCapture, BLESS, XRCC4, and $\gamma-\mathrm{H} 2 \mathrm{AX}$ at predicted DSB regions compared to non-DSB regions over the whole genome. AUROC area under the ROC curve, DSB double-strand break, ROC receiver operating characteristic

controls (Additional file 1: Figure S12c). On average, the END-seq signal accordingly increased with the predicted DSB signal (Additional file 1: Figure S12d). We also tested whether our predictions in MCF-7 cells overlapped etoposide (ETO) induced DSBs mapped by END-seq. Interestingly, we found a strong enrichment of ETO END-seq reads at predicted DSBs compared to predicted controls (Additional file 1: Figure S12e). On average, the END-seq signal accordingly increased with the predicted DSB signal (Additional file 1: Figure S12f).

All these results revealed that the strongest predictors including DNase and CTCF were the same in two different cell types, and that accordingly, a random forest learned in one cell type can efficiently predict DSBs in another cell type.

\section{Prediction from DNA motifs and shape}

We then explored the possibility of predicting DSBs based on DNA sequence using DNA motif occurrences. We built a random forest classifier using 454 available motifs from the JASPAR 2016 database and obtained good prediction accuracy (AUROC $=0.827 ; \mathrm{CI}_{95 \%}:[0.819,0.831]$; AUPR $=0.910$; Fig. 7a; Additional file 1: Figure S13a). Several motifs from the transcription factor complex AP-1 were good predictors, such as FOS::JUN $(\mathrm{VI}=0.016)$ and FOS $(\mathrm{VI}=0.009)$ (Fig. $7 \mathrm{~b})$, which were previously shown to be enriched at DSB sites (see Section "Results and dis cussion", DSBs are enriched with epigenome marks and DNA motifs). Using lasso regression, we improved previous predictions $\left(\mathrm{AUROC}=0.839 ; \mathrm{CI}_{95 \%}\right.$ : $[0.829,0.840]$; AUPR $=0.919$; Fig. 7a; Additional file 1: Figure S13a). Based on lasso regression, we found that the CTCF motif had the highest beta coefficient $(\beta=3.22)$, corresponding to $\mathrm{OR}=25$ (Fig. 7c), supporting recent evidence showing that long-range contacts are involved in DNA repair $[25,35,41]$. Furthermore, motifs of tumor proteins p53, $\mathrm{p} 63$, and $\mathrm{p} 73$ had high coefficients $(\beta>2.03$, OR $>$ 7.6), in agreement with previous predictions based on 
a Predictions from DNA motifs

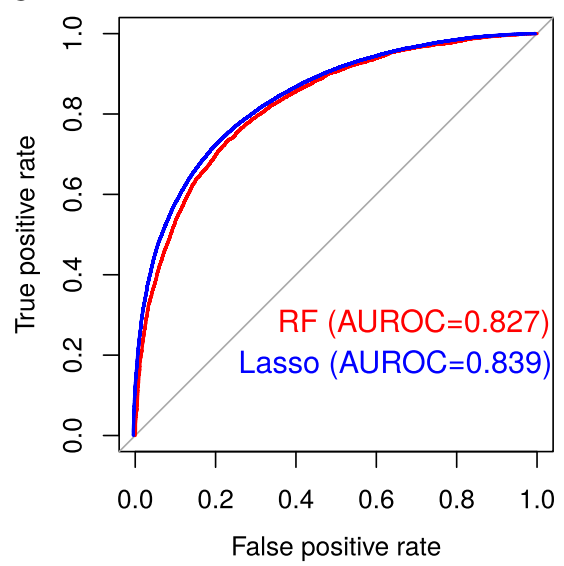

C Lasso regression coefficients

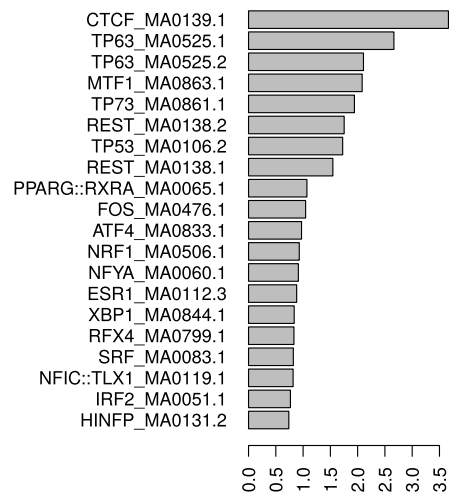

Lasso coefficient
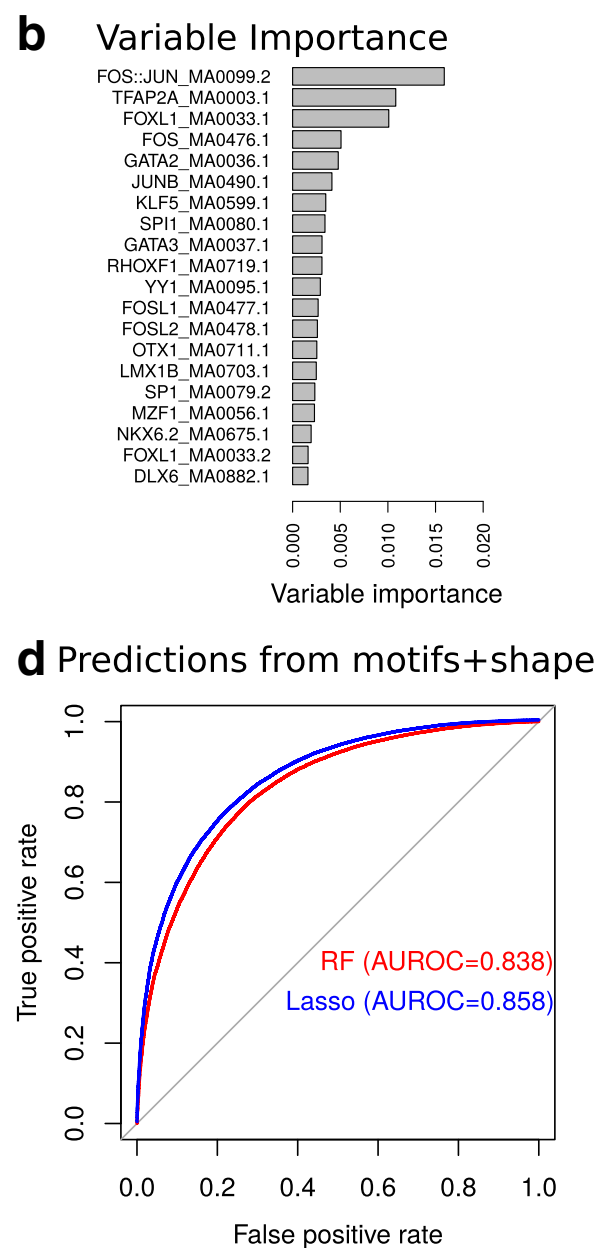

Fig. 7 Prediction of double-strand breaks (DSBs) using DNA motifs and shape. a Receiver operating characteristic (ROC) curve for the DSB predictions using DNA motifs from the JASPAR 2016 database. Random forest (RF) and lasso logistic regression were compared. $\mathbf{b}$ The 20 highest DNA motif variable importance values. c The 20 highest DNA motif lasso coefficients. $\mathbf{d}$ ROC curve for the DSB predictions using DNA motifs with DNA shape. AUROC area under the ROC curve, DSB double-strand break, RF random forest, ROC receiver operating characteristic

ChIP-seq data (see above). We also found motifs recognized by factors involved in heavy metal response (MTF-1: $\beta=2.08$, OR $=8$ ), in oxidative stress response (NRF1: $\beta=0.93$, OR $=2.53$; REST: $\beta=1.75$, OR $=$ 5.75), in endoplasmic reticulum stress (ATF4: $\beta=0.97$, $\mathrm{OR}=2.64)$, and in estrogen-induced DNA damage (ESR1: $\beta=0.88, \mathrm{OR}=2.41$ ). To assess the significance of those motifs, we built a logistic regression model without any regularization including all motifs with $\beta>0.5$. We found that most motifs $(22 / 29)$ were significantly associated with DSBs $(p<0.05$ after false discovery correction; Additional file 1: Table S2). Many of the above mentioned proteins have been shown to interact with each other. For instance, NRF1 associates with Jun proteins of the AP-1 complex [42]. ESR1 associates with AP-1/JUN and FOS to mediate estrogen element response-independent signaling [43].
DNA shape was recently shown to predict transcription factor binding sites and gene expression [14, 44]. Thus, we assessed if DNA shape could similarly serve to predict DSBs together with motifs. For this, we predicted four DNA shape features using simulations: minor groove width (MGW), propeller twist (ProT), roll (Roll), and helix twist (HelT) of DSB sites at base resolution. From each feature, we computed 12 predictors including quantiles $(0,10,20,30,40,50,60,70,80,90$, and $100 \%$ and the variance to describe the distribution of the feature within a DSB site. We used the resulting 48 variables combined with motif occurrences to predict DSBs with random forests and obtained better accuracy (AUROC $=0.838$ and AUPR $=0.915$; Fig. $7 d$; Additional file 1: Figure $\mathrm{S} 13 \mathrm{~b})$ compared to using motifs alone (AUROC $=0.827$ and AUPR $=0.910$; Fig. 7a; Additional file 1: Figure S13a). Among the DNA shape variables, 
ProT median and MGW variance had the highest variable importance ( $\mathrm{VI}=0.01$ and $\mathrm{VI}=0.01$, respectively). Using lasso regression, we also obtained better predictions (AUROC $=0.858$ ), compared to using motifs only (AUROC $=0.839$ and AUPR $=0.928$; Fig. 7d; Additional file 1: Figure S13b). These results reflect the importance of DNA shape in determining DSB sites, in agreement with studies showing that narrow minor grooves (created by either sequence context or DNA bending) limit access of reactive oxygen species [45].

\section{Conclusions}

DSBs are a major threat to a cell and they are associated with cancer development. Over the past years, new techniques have been developed to map DSBs at high resolution and genome-wide level. However, these techniques are costly and challenging. Here, we show, for the first time, that such DSBs can be computationally predicted using public epigenomic data, even when the availability of data is limited (e.g., DNase I and H3K4me1). By using state-of-the-art computational models, we achieve excellent prediction accuracy, paving the way for a better understanding of DSB formation depending on developmental stage or cell-type specific epigenetic marks. Thus, our computational approach should allow the genomewide mapping of DSBs in numerous cell lines and tissues using the ENCODE and Roadmap Epigenomics databases.

There are multiple perspectives for this work. Recent developments from deep (convolutional) neural networks $[13,46]$ can improve model predictions and decrease the number of false positives at the genome level. In addition, our current model did not account for the impact of copy number variation in cancer cells on prediction, and future studies should integrate copy number variation as a quantitative predictor variable in the model to correct for this bias.

\section{Methods}

\section{Double-strand breaks}

All double-strand DNA break data used are summarized in Table 1. We used double-strand DNA breaks mapped by DSBCapture and BLESS in human epidermal keratinocyte (NHEK) cells from the Gene Expression Omnibus (GEO) accession GSE78172 [6]. DSBCapture and BLESS peaks were called using MACS 2.1.0 on human genome assembly hg19 (https:/github.com/ taoliu/MACS). The peaks obtained from two biological replicates were intersected to yield more reliable DSB sites for model predictions.

We used double-strand DNA breaks mapped by DSBCapture and BLESS in AID-DIvA cells, a U2OS cell line (human bone osteosarcoma epithelial cells) expressing the AsiSI restriction enzyme fused to a modified estrogen receptor ligand-binding domain [38]. Upon tamoxifen treatment, AsiSI induces sequence-specific DSBs at GCGATCGC sites. DSBCapture data were from tamoxifen-treated cells from GEO accession GSE78172 [6]. DSBCapture peaks were called using MACS 2.1.0 on human genome assembly hg19. BLESS data were from untreated cells arrested in G1 phase from ArrayExpress accession E-MTAB-4846 [37]. Because of the low coverage of BLESS data, a sufficient number of DSB peaks could not be called.

We used double-strand DNA breaks mapped by BLISS in KBM7 cells (human myeloid leukemia) from NCBI Sequence Read Archive at SRP099132 [40]. We also used double-strand DNA breaks mapped by END-seq in untreated and etoposide-treated MCF-7 cells (human breast cancer) from GSE99197 [35].

\section{ChIP-seq and DNase-seq data}

All ChIP-seq and DNase-seq data used are summarized in Table 2. We used ChIP-seq uniform peaks (CTCF, POL2B, EZH2, H3K4me1/me2/me3, H3K9me1/me3/ac, H3K27me3/ac, H3K36me3, H3K79me2, H4K20me1, and H2A.Z) and DNase-seq uniform peaks for NHEKs from the ENCODE project [7] (https://genome.ucsc.edu/ encode). We also used p63 ChIP-seq of keratinocytes from GEO accession GSE59827 [21].

For U2OS cells, we used DNase-seq and H3K27ac ChIPseq peaks from GEO accession GSE87831 [47]. We used H3K4me1 and POL2B ChIP-seq peaks from GEO accession GSE73742 [48]. We used H3K4me3 and H3K27me3 ChIP-seq peaks from GSE35573 [49]. We used H3K9me3

Table 1 Double-strand DNA break data summary

\begin{tabular}{|c|c|c|c|c|}
\hline Cell line & Treatment & Technique & Number of replicates & Accession \\
\hline NHEK & No treatment & DSBCapture & 2 & GSE78172 \\
\hline NHEK & No treatment & BLESS & 2 & GSE78172 \\
\hline U2OS & 4-hydroxytamoxifen & DSBCapture & 1 & GSE78172 \\
\hline U2OS & No treatment & BLESS & 1 & E-MTAB-4846 \\
\hline KBM7 & No treatment & BLISS & 1 & SRP099132 \\
\hline MCF-7 & No treatment & END-seq & 1 & GSE99197 \\
\hline MCF-7 & Etoposide & END-seq & 1 & GSE99197 \\
\hline
\end{tabular}


Table 2 ChIP-seq and DNase-seq data summary

\begin{tabular}{|c|c|c|c|c|}
\hline Cell line & Treatment & Technique & Number of replicates & Accession \\
\hline NHEK & No treatment & $\begin{array}{l}\text { CTCF, H3K4me3, H3K27me3, } \\
\text { H3K36me3 ChIP-seq }\end{array}$ & 2 & ENCODE uniform peaks \\
\hline NHEK & No treatment & $\begin{array}{lr}\text { EZH2, } & \text { H3K4me1/me2, } \\
\text { H3K9me1/me3/ac, H3K79me2, } & \text { H3K2Ome1, H2A.Z, H3K27ac, POL2B } \\
\text { H4K20 } & \\
\text { ChIP-seq } & \end{array}$ & 1 & ENCODE uniform peaks \\
\hline NHEK & No treatment & DNase-seq & 2 & ENCODE uniform peaks \\
\hline NHEK & No treatment & p63 ChIP-seq & 1 & GSE59827 \\
\hline U2OS & No treatment & DNase-seq, H3K27ac ChIP-seq & 1 & GSE87831 \\
\hline U2OS & No treatment & H3K4me1, POL2B ChIP-seq & 1 & GSE73742 \\
\hline U2OS & No treatment & H3K4me3, H3K27me3 ChIP-seq & 1 & GSE35573 \\
\hline U2OS & No treatment & H3K9me3, H3K36me3 ChIP-seq & 1 & ENCODE \\
\hline U2OS & No treatment & CTCF ChIP-seq & 1 & ChIP-Atlas \\
\hline U2OS & 4-hydroxytamoxifen & XRCC $4, \gamma$-H2A.X ChIP-seq & 1 & E-MTAB-1241 \\
\hline KBM7 & No treatment & DNase-seq & 1 & ChIP-Atlas \\
\hline KBM7 & No treatment & H3K9me3 ChIP-seq & 1 & GSE60056 \\
\hline K562 & No treatment & CTCF, H3K4me1/me3 ChIP-seq & 1 & ENCODE \\
\hline MCF-7 & No treatment & $\begin{array}{l}\text { H3K4me1/me3, H3K9ac/me3, } \\
\text { H3K27me3 ChIP-seq }\end{array}$ & 1 & GSE23701 \\
\hline MCF-7 & No treatment & DNase-seq and CTCF ChIP-seq & 1 & ENCODE \\
\hline
\end{tabular}

and H3K36me3 ChIP-seq peaks from ENCODE [7]. We used CTCF ChIP-seq peaks from the ChIP-Atlas database (http://chip-atlas.org/). We used XRCC4 and $\gamma$-H2A.X ChIP-seq for tamoxifen-treated DIvA cells from ArrayExpress accession E-MTAB-1241 [37].

For KBM7 cells, we used DNase-seq from the ChIPAtlas database, and H3K9me3 ChIP-seq from GSE60056 [50]. Instead of KBM7, we used K562 (chronic myelogenous leukemia) for CTCF, H3K4me1/me3 ChIP-seq from the ENCODE project [7] (https://genome.ucsc. edu/encode). For MCF-7 cells, we used H3K4me1/me3, $\mathrm{H} 3 \mathrm{~K} 9 \mathrm{ac} / \mathrm{me} 3$, and H3K27me3 ChIP-seq without treatment (DMSO) from GSE23701 [51, 52]. We used DNaseseq and CTCF ChIP-seq from ENCODE [7].

\section{DNA motifs}

We used motif position frequency matrices for transcription factor binding sites from the JASPAR 2016 database (http://jaspar.genereg.net). We called transcription factor binding sites over the human genome using the position weight matrices and a minimum matching score of $80 \%$.

\section{DNA shape}

We predicted four DNA shape features using Monte Carlo simulations: minor groove width (MGW) and propeller twist (ProT) at base pair resolution and roll (Roll) and helix twist (HelT) at base pair step resolution using R package DNAshapeR (https://bioconductor.org/ packages/release/bioc/html/DNAshapeR.html).

\section{Random forest and lasso regression}

We used $\mathrm{R}$ package ranger (https://cran.r-project.org/ web/packages/ranger) to compute the random forest classification efficiently [30]. We used the default package parameters: num.trees $=500$ and mtry is the square root of the number of variables. Variable importance was computed using the mean decrease in accuracy in the out-of-bag sample. To discriminate between DSB and non-DSB sites, we randomly selected genomic sequences that matched sizes, GC, and repeat contents of DSB sites using $\mathrm{R}$ package gkmSVM (https://cran.r-project. org/web/packages/gkmSVM). To learn the model, we mapped epigenomic data, DNA motifs, and DNA shape as follows. For epigenomic data including ChIP-seq and DNase-seq data, we used peak genomic coordinates of a feature (for instance, CTCF binding sites) and considered the presence $(x=1)$ or absence $(x=0)$ of the corresponding feature at the DSB site. If a feature peak overlapped only $60 \%$ of the DSB site, then $x=0.6$. For DNA motifs, we computed the number of motif occurrences within DSB and non-DSB sites. For DNA shape, we computed four features including MGW, ProT, Roll, and HelT of DSB sites at base resolution. For each DNA shape feature, we then computed 12 predictors, including quantiles $(0,10,20,30,40,50,60,70,80,90$, and $100 \%)$ and the variance to describe the distribution of the feature within a DSB site. The DSB data were next split into two sets: the training set used for learning the model and a test set used for assessing prediction 
accuracy. We also used $\mathrm{R}$ package glmnet (https://cran. r-project.org/web/packages/glmnet/index.html) to compute lasso logistic regression with cross-validation. To assess the prediction accuracy of random forest and lasso regression, we computed the ROC curve and AUROC. To estimate the confidence interval for AUROC, we used the pROC R package (https://cran.r-project.org/ web/packages/pROC). We also computed the PR curve and AUPR to assess prediction accuracy when the classes were very imbalanced, especially for genome-wide analyses. For this, we used the PRROC R package (https://cran. r-project.org/web/packages/PRROC).

\section{Additional file}

Additional file 1: Additional figures and tables. Figures S1-13 and Tables S1, S2. (PDF $1618 \mathrm{~kb}$ )

\section{Acknowledgments}

The authors are grateful to the Balasubramanian lab (Babraham Institute, UK), to the Crosetto lab (Karolinska Institutet, Sweden), and to the Nussenzweig lab (National Institutes of Health, USA) for data and for help in processing the data.

\section{Funding}

This work was supported by the University of Toulouse and by the CNRS. Funding for open access charge: Fondation pour la Recherche Médicale (DEQ20160334940).

\section{Availability of data and materials}

The pipeline was developed in the $\mathrm{R}$ language and is available at https://github.com/morphos30/PredDSB [53] under Apache License 2.0. The v1.0 release was deposited at https://zenodo.org/badge/latestdoi/117546880 with DOI 10.5281/zenodo.1174011.

The data used in this study were downloaded using the following accession numbers and databases:

- GSE78172 (NHEK DSBCapture and BLESS) [6]

- GSE78172 (U2OS AID-DlvA DSBCapture) [6]

- E-MTAB-4846 (U2OS AID-DIVA BLESS) [37]

- SRP099132 (KBM7 BLISS) [40]

- GSE99197 (MCF-7 END-seq) [35]

- ENCODE (NHEK ChIP-seq and DNase-seq) [7]

- GSE59827 (NHEK p63 ChIP-seq) [21]

- GSE87831 (U2OS DNase-seq and H3K27ac ChIP-seq) [47]

- GSE73742 (U2OS H3K4me1 and POL2B ChIP-seq) [48]

- GSE35573 (U2OS H3K4me3 and H3K27me3 ChIP-seq) [49]

- ENCODE (U2OS H3K9me3 and H3K36me3 ChIP-seq) [7]

- ChIP-Atlas database (U2OS CTCF ChIP-seq) [54]

- E-MTAB-1241 (U2OS XRCC4 and $\gamma$-H2A.X ChIP-seq) [37]

- ChIP-Atlas database (KBM7 DNase-seq) [54]

- GSE60056 (KBM7 H3K9me3 ChIP-seq) [50]

- ENCODE (K562 CTCF and H3K4me1/me3 ChIP-seq) [7]

- GSE23701 (MCF-7H3K4me1/me3,H3K9ac/me3,H3K27me3ChIP-seq)[51, 52]

- ENCODE (MCF-7 DNase-seq and CTCF ChIP-seq) [7].

\section{Authors' contributions}

RM supervised the project, conceived the method, wrote the code, designed the data analysis, and analyzed the data. KG performed the BLESS experiments for U2OS AID-DIVA cells. RM, GL, and OC interpreted the results and wrote the paper. All authors read and approved the final manuscript.

\section{Ethics approval and consent to participate}

Not applicable.

\section{Consent for publication}

Not applicable.
Competing interests

The authors declare that they have no competing interests.

\section{Publisher's Note}

Springer Nature remains neutral with regard to jurisdictional claims in published maps and institutional affiliations.

\section{Author details}

${ }^{1}$ LBME, Centre de Biologie Intégrative (CBI), Université de Toulouse, CNRS, UPS, 118 , route de Narbonne, 31062 Toulouse, France. ${ }^{2}$ Laboratory of

Bioinformatics and Systems Biology, Centre of New Technologies, University of Warsaw, Zwirki i Wigury 93, 02-089 Warsaw, Poland. ${ }^{3}$ LBCMCP, Centre de Biologie Intégrative (CBI), Université de Toulouse, CNRS, UPS, 118, route de Narbonne, 31062 Toulouse, France.

Received: 30 October 2017 Accepted: 22 February 2018

Published online: 15 March 2018

\section{References}

1. McKinnon PJ, Caldecott KW. DNA strand break repair and human genetic disease. Annu Rev Genomics Hum Genet. 2007:8(1):37-55. https://doi. org/10.1146/annurev.genom.7.080505.115648.

2. Mehta A, Haber JE. Sources of DNA double-strand breaks and models of recombinational DNA repair. Cold Spring Harb Perspect Biol. 2014;6(9): 016428. https://doi.org/10.1101/cshperspect.a016428. http://cshperspectives.cshlp.org/content/6/9/a016428.full.pdf+html.

3. Crosetto N, Mitra A, Silva MJ, Bienko M, Dojer N, Wang Q, et al. Nucleotide-resolution DNA double-strand break mapping by next-generation sequencing. Nat Methods. 2013;10(4):361-5. https://doi. org/10.1038/nmeth.2408

4. Tsai SQ, Zheng Z, Nguyen NT, Liebers M, Topkar W, Thapar V, et al. GUIDE-seq enables genome-wide profiling of off-target cleavage by CRISPR-Cas nucleases. Nat Biotechnol. 2015;33(2):187-97.

5. Canela A, Sridharan S, Sciascia N, Tubbs A, Meltzer P, Sleckman B, et al. DNA breaks and end resection measured genome-wide by end sequencing. Mol Cell. 2016;63(5):898-911.

6. Lensing SV, Marsico G, Hansel-Hertsch R, Lam EY, Tannahill D, Balasubramanian S. DSBCapture: in situ capture and sequencing of DNA breaks. Nat Methods. 2016;13(10):855-7. https://doi.org/10.1038/nmeth.3960.

7. The ENCODE Consortium. An integrated encyclopedia of DNA elements in the human genome. Nature. 2012;489(7414):57-74. https://doi.org/10, 1038/nature11247.

8. The Roadmap Epigenomics Consortium, Kundaje A, Meuleman W, Ernst J, Bilenky M, Yen A, Heravi-Moussavi A, et al. Integrative analysis of 111 reference human epigenomes. Nature. 2015;518(7539):317-30. https://doi.org/10.1038/nature14248.

9. Kleftogiannis D, Kalnis P, Bajic VB. DEEP: a general computational framework for predicting enhancers. Nucleic Acids Res. 2014;43(1):6. https://doi.org/10.1093/nar/gku1058.

10. Ernst J, Kellis M. ChromHMM: automating chromatin-state discovery and characterization. Nat Methods. 2012;9(3):215-6. https://doi.org/10.1038/ nmeth.1906.

11. Taverna SD, Li H, Ruthenburg AJ, Allis CD, Patel DJ. How chromatin-binding modules interpret histone modifications: lessons from professional pocket pickers. Nat Struct Mol Biol. 2007;14(11):1025-40. https://doi.org/10.1038/nsmb1338.

12. Whitaker JW, Chen Z, Wang W. Predicting the human epigenome from DNA motifs. Nat Methods. 2015;12(3):265-72.

13. Zhou J, Troyanskaya OG. Predicting effects of noncoding variants with deep learning-based sequence model. Nat Methods. 2015;12(10):931-4. https://doi.org/10.1038/nmeth.3547.

14. Mathelier A, Xin B, Chiu TP, Yang L, Rohs R, Wasserman WW. DNA shape features improve transcription factor binding site predictions in vivo. Cell Syst. 2016;3(3):278-864. https://doi.org/10.1016/j.cels.2016.07.001.

15. Hayashi K, Yoshida K, Matsui Y. A histone H3 methyltransferase controls epigenetic events required for meiotic prophase. Nature. 2005;438(7066): 374-8. https://doi.org/10.1038/nature04112.

16. Myers S, Bowden R, Tumian A, Bontrop RE, Freeman C, MacFie TS. Drive against hotspot motifs in primates implicates the PRDM9 gene in meiotic recombination. Science. 2010;327(5967):876-9. https://doi.org/10.1126/ 
science.1182363. http://science.sciencemag.org/content/327/5967/876. full.pdf.

17. Baudat F, Buard J, Grey C, Fledel-Alon A, Ober C, Przeworski M. PRDM9 is a major determinant of meiotic recombination hotspots in humans and mice. Science. 2010;327(5967):836-40. https://doi.org/10.1126/science. 1183439. http://science.sciencemag.org/content/327/5967/836.full.pdf.

18. Kinner A, Wu W, Staudt C, lliakis G. $\gamma$-H2AX in recognition and signaling of DNA double-strand breaks in the context of chromatin. Nucleic Acids Res. 2008;36(17):5678-94. https://doi.org/10.1093/nar/gkn550.

19. Price $B D, D^{\prime} A n d r e a A D$. Chromatin remodeling at DNA double-strand breaks Cell. 2013;152(6):1344-54. https://doi.org/10.1016/j.cell.2013.02.011.

20. Ghandi M, Mohammad-Noori M, Ghareghani N, Lee D, Garraway L, Beer MA. gkmSVM: an R package for gapped-kmer SVM. Bioinformatics. 2016;32(14):2205-7. https://doi.org/10.1093/bioinformatics/btw203.

21. Kouwenhoven EN, Oti M, Niehues $H$, van Heeringen SJ, Schalkwijk J, Stunnenberg HG, et al. Transcription factor p63 bookmarks and regulates dynamic enhancers during epidermal differentiation. EMBO Rep. 2015;16(7):863-78. https://doi.org/10.15252/embr.201439941.

22. Mathelier A, Fornes O, Arenillas DJ, Chen C-Y, Denay G, Lee J, et al. JASPAR 2016: a major expansion and update of the open-access database of transcription factor binding profiles. Nucleic Acids Res. 2016;44(D1):110-5. https://doi.org/10.1093/nar/gkv1176.

23. Chiu TP, Comoglio F, Zhou T, Yang L, Paro R, Rohs R. DNAshapeR: an R/Bioconductor package for DNA shape prediction and feature encoding. Bioinformatics. 2016;32(8):1211-3. https://doi.org/10.1093/ bioinformatics/btv735.

24. Tibshirani R. Regression shrinkage and selection via the lasso. J R Stat Soc Ser B (Methodol). 1996;58(1):267-88. https://doi.org/10.2307/2346178.

25. Tchurikov NA, Fedoseeva DM, Sosin DV, Snezhkina AV, Melnikova NV, Kudryavtseva AV, et al. Hot spots of DNA double-strand breaks and genomic contacts of human rDNA units are involved in epigenetic regulation. J Mol Cell Biol. 2015;7(4):366-82. https://doi.org/10.1093/ jmcb/mju038.

26. Caron P, Aymard F, lacovoni JS, Briois $S$, Canitrot $Y$, Bugler $B$, et al. Cohesin protects genes against $\gamma-\mathrm{H} 2 \mathrm{AX}$ induced by DNA double-strand breaks. PLoS Genet. 2012;8(1):10002460. https://doi.org/10.1371/journal. pgen.1002460.

27. Phillips-Cremins JE, Sauria MEG, Sanyal A, Gerasimova TI, Lajoie BR, Bell JSK, et al. Architectural protein subclasses shape 3D organization of genomes during lineage commitment. Cell. 2013;153(6):1281-95. https://doi.org/10.1016/j.cell.2013.04.053.

28. Lin YL, Sengupta S, Gurdziel K, Bell GW, Jacks T, Flores ER. p63 and p73 transcriptionally regulate genes involved in DNA repair. PLOS Genet. 2009;5(10):1000680. https://doi.org/10.1371/journal.pgen.1000680.

29. Williams AB, Schumacher B. p53 in the DNA-damage-repair process. Cold Spring Harb Perspect Med. 2016;6(5):026070. https://doi.org/10.1101/ cshperspect.a026070. http://perspectivesinmedicine.cshlp.org/content/ 6/5/a026070.full.pdf+html.

30. Breiman L. Random forests. Mach Learn. 2001;45(1):5-32. https://doi.org/ 10.1023/A:1010933404324.

31. Jacquet K, Fradet-Turcotte A, Avvakumov N, Lambert JP, Roques $C$, Pandita R, et al. The TIP60 complex regulates bivalent chromatin recognition by 53BP1 through direct H4K20me binding and H2AK15 acetylation. Mol Cell. 2016;62(3):409-21. https://doi.org/10.1016/j.molcel. 2016.03.031.

32. Tjeertes JV, Miller KM, Jackson SP. Screen for DNA-damage-responsive histone modifications identifies H3K9Ac and H3K56Ac in human cells. EMBO J. 2009;28(13):1878-89. https://doi.org/10.1038/emboj.2009.119. http://emboj.embopress.org/content/28/13/1878.full.pdf.

33. Lhoumaud P, Hennion M, Gamot A, Cuddapah S, Queille S, Liang J, et al. Insulators recruit histone methyltransferase $\mathrm{dMes} 4$ to regulate chromatin of flanking genes. EMBO J. 2014;33(14):1599-613. https://doi.org/10.15252/embj.201385965.

34. Pfister SX, Ahrabi S, Zalmas LP, Sarkar S, Aymard F, Bachrati CZ, et al. SETD2-dependent histone H3K36 trimethylation is required for homologous recombination repair and genome stability. Cell Rep. 2014;7(6):2006-18. https://doi.org/10.1016/j.celrep.2014.05.026.

35. Canela A, Maman Y, Jung S, Wong N, Callen E, Day A, et al. Genome organization drives chromosome fragility. Cell. 2017;170(3):507-2118. https://doi.org/10.1016/j.cell.2017.06.034.
36. Hilmi K, Jangal M, Marques M, Zhao T, Saad A, Zhang C, et al. CTCF facilitates DNA double-strand break repair by enhancing homologous recombination repair. Sci Adv. 2017;3(5):1601898. https://doi.org/10. 1126/sciadv.1601898. http://advances.sciencemag.org/content/3/5/ e1601898.full.pdf.

37. Aymard F, Aguirrebengoa M, Guillou E, Javierre BM, Bugler B, Arnould $C$, et al. Genome-wide mapping of long-range contacts unveils clustering of DNA double-strand breaks at damaged active genes. Nat Struct Mol Biol. 2017;24(4):353-61. https://doi.org/10.1038/nsmb.3387.

38. Iacovoni JS, Caron P, Lassadi I, Nicolas E, Massip L, Trouche D, et al. High-resolution profiling of $\gamma-\mathrm{H} 2 \mathrm{AX}$ around DNA double strand breaks in the mammalian genome. EMBO J. 2010;29(8):1446-57. https://doi.org/10. 1038/emboj.2010.38. http://emboj.embopress.org/content/29/8/1446. full.pdf.

39. Savic V, Yin B, Maas NL, Bredemeyer AL, Carpenter AC, Helmink BA, et al. Formation of dynamic $\gamma-\mathrm{H} 2 \mathrm{AX}$ domains along broken DNA strands is distinctly regulated by ATM and MDC1 and dependent upon H2AX densities in chromatin. Mol Cell. 2009;34(3):298-310. https://doi.org/10. 1016/j.molcel.2009.04.012.

40. Yan WX, Mirzazadeh R, GarneroneS, ScottD, Schneider MW, Kallas T, et al. BLISS is a versatile and quantitative method for genome-wide profiling of DNA double-strand breaks. Nat Commun. 2017;8:15058. https://doi.org/ 10.1038/ncomms15058.

41. Bekker-Jensen S, Mailand N. Assembly and function of DNA double-strand break repair foci in mammalian cells. DNA Repair. 2010;9(12):1219-28. https://doi.org/10.1016/j.dnarep.2010.09.010.

42. Venugopal R, Jaiswal AK. Nrf2 and Nrf1 in association with Jun proteins regulate antioxidant response element-mediated expression and coordinated induction of genes encoding detoxifying enzymes. Oncogene. 1998;17(24):3145-56.

43. Kushner PJ, Agard DA, Greene GL, Scanlan TS, Shiau AK, Uht RM, et al. Estrogen receptor pathways to AP-1. J Steroid Biochem Mol Biol. 2000;74(5):311-7.

44. Peng PC, Sinha S. Quantitative modeling of gene expression using DNA shape features of binding sites. Nucleic Acids Res. 2016;44(13):120. https://doi.org/10.1093/nar/gkw446.

45. Cannan WJ, Pederson DS. Mechanisms and consequences of double-strand DNA break formation in chromatin. J Cell Physiol. 2016;231(1):3-14. https://doi.org/10.1002/jcp.25048.

46. Kim SG, Harwani M, Grama A, Chaterji S. EP-DNN: a deep neural network-based global enhancer prediction algorithm. Sci Rep. 2016;6:38433.

47. Ibarra A, Benner C, Tyagi S, Cool J, Hetzer MW. Nucleoporin-mediated regulation of cell identity genes. Gene Dev. 2016;30(20):2253-8. https://doi.org/10.1101/gad.287417.116.

48. Pradhan SK, SuT, Yen L, Jacquet K, Huang C, Cote J, et al. EP400 deposits H3.3 into promoters and enhancers during gene activation. Mol Cell. 2016;61 (1):27-38. https://doi.org/10.1016/j.molcel.2015.10.039.

49. Easwaran H, Johnstone SE, Van Neste L, Ohm J, Mosbruger T, Wang Q, et al. A DNA hypermethylation module for the stem/progenitor cell signature of cancer. Genome Res. 2012;22(5):837-49. https://doi.org/10. 1101/gr.131169.111.

50. Tchasovnikarova IA, Timms RT, Matheson NJ, Wals K, Antrobus R, Göttgens B. Epigenetic silencing by the HUSH complex mediates position -effect variegation in human cells. Science. 2015;348(6242):1481-5. https://doi.org/10.1126/science.aaa7227.

51. Joseph R, Orlov YL, Huss M, Sun W, Li Kong S, Ukil L. Integrative model of genomic factors for determining binding site selection by estrogen receptor- $\alpha$. Mol Syst Biol. 2010;6:456. https://doi.org/10.1038/msb.2010.109.

52. Kong SL, Li G, Loh SL, Sung WK, Liu ET. Cellular reprogramming by the conjoint action of ER $\alpha, F O X A 1$, and GATA3 to a ligand-inducible growth state. Mol Syst Biol. 2011;7:526. https://doi.org/10.1038/msb.2011.59.

53. Mourad R. morphos30/preddsb v1.0. GitHub. 2018. https://doi.org/10. 5281/zenodo.1174011. https://github.com/morphos30/PredDSB.

54. Oki S, Ohta T, Shioi G, Hatanaka H, Ogasawara O, Okuda Y, et al. Integrative analysis of transcription factor occupancy at enhancers and disease risk loci in noncoding genomic regions. bioRxiv. 2018:262899. https://doi.org/10.1101/262899. 